\title{
Computational Difficulty of Finding Matrix Product Ground States
}

\author{
Norbert Schuch, ${ }^{1}$ Ignacio Cirac, ${ }^{1}$ and Frank Verstraete ${ }^{2}$ \\ ${ }^{1}$ Max-Planck-Institut für Quantenoptik, Hans-Kopfermann-Strasse 1, D-85748 Garching, Germany \\ ${ }^{2}$ Fakultät für Physik, Universität Wien, Boltzmanngasse 5, A-1090 Wien, Austria \\ (Received 22 February 2008; revised manuscript received 7 May 2008; published 27 June 2008)
}

\begin{abstract}
We determine the computational difficulty of finding ground states of one-dimensional (1D) Hamiltonians, which are known to be matrix product states (MPS). To this end, we construct a class of 1D frustration-free Hamiltonians with unique MPS ground states and a polynomial gap above, for which finding the ground state is at least as hard as factoring. Without the uniqueness of the ground state, the problem becomes NP complete, and thus for these Hamiltonians it cannot even be certified that the ground state has been found. This poses new bounds on convergence proofs for variational methods that use MPS.
\end{abstract}

Explaining the behavior of correlated quantum manybody systems is one of the major challenges in physics. The exponential dimension of the underlying Hilbert space renders a straightforward numerical simulation impossible with respect to both computational time and storage space. However, for specific physical scenarios simulation methods have been developed; in particular, the density matrix renormalization group (DMRG) method [1,2] has proven extremely successful in describing ground and thermal states of 1D spin systems to very high accuracy.

DMRG has a natural interpretation as a variational ansatz over the class of matrix product states (MPS) [3], and it has been proven that every ground state of a local gapped Hamiltonian can indeed be approximated efficiently by MPS [4]. While this explains why MPS are well suited to describe ground states of 1D quantum systems, essentially nothing fundamental concerning the convergence of the variational method could be shown, despite the fact that in practice it converges extremely well. Actually, given the type of optimization problem, one is rather tempted to believe that DMRG will typically get stuck in local minima, and, in fact, it has been shown that if the optimization is performed in a specific way where sites are optimized simultaneously, configurations might occur where the optimization problem becomes NP hard, meaning the algorithm would get stuck [5]. Yet, this difficulty is solely due to the specific way in which the optimization is performed, rather than being a fundamental problem of any variational method over MPS.

As we show in this Letter, however, under natural assumptions on the Hamiltonian obtaining a good MPS approximation to the ground state, and, in particular, finding the minimum in DMRG, is a computationally hard problem which can take exponential time. More precisely, we construct a class of nearest neighbor Hamiltonians on a 1D chain of length $L$ with the following properties: Any of those Hamiltonians has a unique ground state with a spectral gap of order $1 / \operatorname{poly}(L)$ above, it is frustration-free (i.e., the ground state minimizes each local term of the Hamiltonian), and the ground state is an MPS, as are the low-lying excited states. For these Hamiltonians, we show that finding the ground state (or a polynomial-accuracy approximation thereof) is a hard problem, as it, e.g., encompasses factoring numbers. This implies that an MPS approximation of these ground states most likely cannot be found efficiently by classical computers.

If instead of requiring a unique MPS ground state we allow for a ground state subspace spanned by MPS while keeping the polynomial energy gap, and instead of frustration freeness require the ground states only to be eigenstates to each local term, we obtain a class of Hamiltonians for which finding the ground state is an NP-complete problem. This implies that for this class it is even impossible to certify that the ground state has been found, based on widely believed complexity theoretic assumptions. Moreover, it follows that there cannot even be a DRMGlike algorithm that works at least for frustration-free systems, since this would allow for the efficient solution of NP-complete problems.

Let us first introduce some basic concepts. An MPS on a length $L$ chain of $d$-level systems ("spins") with bond dimension $D$ is given by $|\psi\rangle=$ $\sum_{i_{1}, \ldots, i_{L}} \operatorname{Tr}\left[A_{i_{1}}^{[1]} \ldots A_{i_{L}}^{[L]}\right]\left|i_{1}, \ldots, i_{L}\right\rangle$, where the $A_{i_{k}}^{[k]}$ are $D \times$ $D$ matrices. The classical complexity class NP contains all decision problems where for "yes" instances, an efficiently checkable proof can be found, as, e.g., colorability of a graph. A problem is said to be hard for a class if any problem in this class can be reduced to solving this very problem, and complete if it is additionally inside the class. While NP hardness of a problem strictly speaking does not prove that it cannot be solved efficiently, it is the best we can hope for, given that showing whether $\mathrm{NP} \neq \mathrm{P}$, although generally believed to be true, is one of the most important open questions in complexity theory.

To construct hard ground state problems for DMRG, we start from the local Hamiltonian problem and the corresponding complexity class QMA (quantum Merlin Arthur). QMA contains those decision problems where for "yes" instances, there is a quantum proof that can be checked 
efficiently by a quantum computer, and is thus the natural quantum generalization of NP. More precisely, in the definition of QMA there are thresholds $p>q$ : for "yes" instances, there is a proof that will be accepted with probability at least $p$, whereas for "no" instances, no attempt to provide a fake proof will succeed with probability more than $q$. It is sufficient to require $p-q>$ $1 / \operatorname{poly}(N)$ (with $N$ the problem size), since then the probabilities can be amplified up to exponentially close to 1 and 0 , respectively [6]. As shown by Kitaev [6,7], the problem LOCAL HAMILTONIAN is complete for QMA: Given a local Hamiltonian on $N$ spins (where in this case "local" means it is a sum of few-particle terms), decide whether the ground state energy is below $a$ or above $b$, with $b-a>$ $1 / \operatorname{poly}(N)$. It is easy to see that this problem is in QMAthe proof is the ground state (or several copies thereof), and the verifier estimates the ground state energy by measuring the local terms.

Let us now review Kitaev's construction for proving QMA hardness of LOCAL HAMILTONIAN. The task is, given a polynomial-size quantum circuit (the verifier), to construct a local Hamiltonian for which the ground state energy is at least $1 / \operatorname{poly}(N)$ lower if there exists a satisfying input to the circuit, i.e., a valid proof. To this end, write the verifying circuit using $T=\operatorname{poly}(N)$ one- and two-qubit gates $U_{t}$, and for each valid input $\left|\phi_{0}\right\rangle$ to the circuit construct a state that encodes the history of the verifier checking this very input,

$$
|\psi\rangle=\sum_{t=0}^{T} U_{t} \cdots U_{1}\left|\phi_{0} \otimes 0 \cdots 0\right\rangle_{d}|t\rangle_{t},
$$

where $d$ denotes the data register [initially, the first part holds the input $\left|\phi_{0}\right\rangle$ and the second poly $(N)$ ancillas that are initialized to $|0 \ldots 0\rangle$; it thus consists of $M=\operatorname{poly}(N)$ qubits] and $t$ the time register. Now, construct a Hamiltonian that penalizes wrong proof histories,

$$
H=H_{\text {init }}+H_{\text {evol }}+H_{\text {final }},
$$

where $H_{\text {init }}=T \sum_{a}|1\rangle_{a}\langle 1|\otimes| 0\rangle_{t}\langle 0|$ penalizes any ancilla $a$ that is not properly initialized, $H_{\mathrm{evol}}=\sum_{t}-U_{t}|t\rangle \times$ $\left\langle t-1\left|-U_{t}^{\dagger}\right| t-1\right\rangle\langle t|+| t-1\rangle\langle t-1|+| t\rangle\langle t| \quad$ ensures that the transitions between $t-1$ and $t$ in $|\psi\rangle$ are correct, and $H_{\text {final }}=|0\rangle_{1}\langle 0|\otimes| T\rangle_{t}\langle T|$ penalizes the state $|0\rangle$ on the very first qubit - it will be set to $|1\rangle$ if the circuit accepts the proof. It has been shown that if there exists a proof that will be accepted by the verifier with high probability, the ground state energy of $(2)$ is by $1 / \operatorname{poly}(N)$ lower than if there is no such proof. The intuition is that in the former case, the state (1) almost does the job, while in the latter case, at least one of the terms in the Hamiltonian (or a superposition thereof) has to be violated.

In general, the spectrum of (2) will be complicated since from the definition of QMA, there can be many potential witnesses with different acceptance probabilities. In the following, we will restrict to problems that have classical verifiers that are accepted deterministically, corresponding to problems in the complexity class NP [8], which will simplify the spectrum considerably and will finally give rise to the simple entanglement structure of the ground state we are after.

To determine the spectral properties of (2) for a classical deterministic circuit, let us fix a classical initial state of the data register $|\boldsymbol{a}\rangle_{d}=\left|a_{1} \ldots a_{M}\right\rangle_{d}$ and analyze the system on the $(T+1)$-dimensional space $\mathcal{H}_{a}$ spanned by $\left|\chi_{t}\right\rangle=$ $U_{t} \cdots U_{1}|\boldsymbol{a}\rangle_{d}|t\rangle_{t}$, which is closed under the action of $H_{\text {init }}, H_{\text {final }}$, and $H_{\text {evol }}$. In particular,

$$
H_{a}=\left.H\right|_{\mathcal{H}_{a}}=\left(\begin{array}{ccccc}
T A+1 & -1 & & & \\
-1 & 2 & -1 & & \\
& \ddots & \ddots & \ddots & \\
& & -1 & 2 & -1 \\
& & & -1 & B+1
\end{array}\right)
$$

with $A=0,1,2, \ldots$ the number of wrongly initialized ancillas in $|\boldsymbol{a}\rangle_{d}$ and $B=0,1$ depending on whether the circuit accepts or rejects the input $|\boldsymbol{a}\rangle_{d}$. In the case of a "yes" instance of the NP problem, there exists an $|\boldsymbol{a}\rangle_{d}$ for which $A=B=0$, whereas for "no" instances, the lowest energy subspace has $A=0, B=1$. In both cases, the eigenfunctions are

$$
\left|\psi_{a, n}\right\rangle=C \sum_{t=0}^{T} \cos \left[\omega_{n}\left(t+\frac{1}{2}\right)\right]\left|\chi_{t}\right\rangle, \quad n=0, \ldots, T,
$$

where $\omega_{n} \equiv \omega_{n}^{0}=n \pi /(T+1)$ for $A=0, B=0$ and $\omega_{n} \equiv \omega_{n}^{1}=\left(n+\frac{1}{2}\right) \pi /\left(T+\frac{3}{2}\right)$ for $A=0, B=1$, respectively; in both cases, the eigenvalues are given by $\lambda_{n}=$ $2\left(1-\cos \omega_{n}\right)$, and $C^{2}=\Theta(1 / T)[10]$.

Different from QMA-completeness proofs, we are not interested in the difference in ground state energy between "yes" and "no" instances but rather in the spectral gap for each of the cases independently. Analyzing the spectrum will be simplified a lot by the fact that the subspaces $\mathcal{H}_{a}$ are closed under the action of any term in the Hamiltonian, which is due to the restriction to classical deterministic circuits.

We start by analyzing $H$ for a circuit corresponding to a "yes" instance of the NP problem. Then, there is at least one initial state $\left|\boldsymbol{a}_{0}\right\rangle_{d}$ such that $H_{\boldsymbol{a}_{0}}$ has $A=B=0$ in (3), and since $H \geq 0$, this subspace contains a ground state. There are two different types of excited states: The ones within $\mathcal{H}_{a_{0}}$, which have a gap $2\left(\cos \omega_{0}^{0}-\cos \omega_{1}^{0}\right)=$ $\Omega\left(1 / T^{2}\right)$, and those within another $\mathcal{H}_{a}$ for which $A$ and/ or $B$ are strictly positive. The energy in any of these subspaces is bounded by the ground state energy for $A=$ $0, B=1$, and is thus $\Omega\left(1 / T^{2}\right)$ as well, proving an $\Omega\left(1 / T^{2}\right)$ spectral gap of the overall Hamiltonian. Note that the degeneracy of the ground state manifold equals the number of different accepted inputs. 
On the other hand, for "no" instances there is no subspace with $A=B=0$. It is easily seen that the ground state subspace has $A=0, B=1$, with ground state energy $2\left(1-\cos \omega_{0}^{1}\right)$ and an $\Omega\left(1 / T^{2}\right)$ gap within the subspace. In order to bound the gap to subspaces with $A \geq 1$ (for which $B$ can be 0), we use the following lemma, shown in [7]: Given $P \geq 0, Q \geq 0$ with null eigenspaces,

$$
P+Q \geq \min \{\Delta(P), \Delta(Q)\}(1-\cos \theta),
$$

where $\Delta(O)>0$ is the smallest nonzero eigenvalue of $O$, and $\theta$ the angle between the null spaces of $P$ and $Q$. It follows that the lowest eigenvalue in an $A=1, B=0$ subspace is at least $T\left(1-\cos \omega_{1}^{0}\right)[1-\sqrt{T /(T+1)}]$, and thus any subspace with $A \geq 1$ has an energy $\Omega\left(1 / T^{2}\right)$ above the ground state.

An important point to observe is the particularly simple entanglement structure of the eigenstates (4) of $H$, which in 1D will allow one to represent them as MPS. To see this, take any classical reversible verifying circuit and decompose it into a sequence of local gates. Let us first allow for three-qubit gates, so we can use the Toffoli gate, which is universal for classical reversible computation. Since it is classical, each of the states $|\boldsymbol{a}(t)\rangle=U_{t} \cdots U_{1}|\boldsymbol{a}\rangle_{d}$ is classical itself, and thus each of the eigenstates (4) is a superposition of only $T+1$ classical terms $|\boldsymbol{a}(t)\rangle|t\rangle \equiv\left|\chi_{t}\right\rangle$. As we want to restrict to two-qubit gates, each Toffoli gate is implemented using a short sequence of entangling twoqubits gates. This temporarily creates entanglement between the three neighboring qubits on which the Toffoli is applied, thus adding some entanglement to the eigenstates. However, this entanglement is both spatially and "temporally" restricted, since it only involves three qubits and it only persists over a few time steps.

Let us now turn towards 1D systems. We will employ the QMA-complete Hamiltonian construction of Aharonov et al. [11], where the time is encoded in the position of the data register. To this end, the data register is realized sequentially $T+1$ times in the $1 \mathrm{D}$ system [which thus consists of $L=M(T+1)=\operatorname{poly}(N)$ sites], and with each time step, the active register moves to the right. To mark which is the active register and to implement a Hamiltonian (2), a control register is appended to each qubit. It is used both to store the status of the register (i.e., used, active, or unused) and to implement an involved scheme in which a head is moving back and forth, thereby first implementing the desired operation $U_{t}$ on the active register and then, qubit by qubit, copying it to the next time slice. Thereby, each original $t$-time step is encoded in $K=$ $O\left(M^{2}\right)$ elementary movements of the head (" $\tau$-time steps"), replacing the original $T$ steps by $\mathcal{T}=K T=$ $\operatorname{poly}(N)$ steps of the encoded system. The resulting local dimension per site is 12 , and the resulting Hamiltonian acts on nearest neighbors only.

As before, the Hamiltonian is a sum of transition rules $\tilde{H}_{\text {evol }}$ (now encoding the elementary movements of the head) and of penalties $\tilde{H}_{\text {init }}$ and $\tilde{H}_{\text {final }}$ for undesired initial and final configurations, acting on the blocks corresponding to $t=0$ and $t=T$, which are applied when the head moves over the qubit. Additionally, one now has to make sure the system stays in the subspace of allowed configurations of the status register, excluding, e.g., the occurrence of more than one head. This is achieved by adding a sum of local penalty terms $\tilde{H}_{\text {penalty }}$ acting on the status register, which either penalize forbidden configurations directly or indirectly as they evolve to penalized ones under $\tilde{H}_{\text {evol }}$. (For details, see [11].)

To analyze the spectral properties of the 1D Hamiltonian, we apply the "clairvoyance lemma" of Ref. [11], which tells us that we can restrict our attention to the subspace of valid configurations of the status register. To this end, split the total Hilbert space into subspaces $\mathcal{K}_{S}$ spanned by minimal sets of classical status register configurations $\mathcal{S}$ closed under $\tilde{H}_{\text {evol }}$; data degrees of freedom are left unrestricted. By definition, these subspaces are also closed under $\tilde{H}_{\text {penalty }}$. There is one subspace $\mathcal{K}_{0}$ that contains only valid configurations, whereas all other $\mathcal{K}_{S}$ contain only illegal configurations. The clairvoyance lemma shows that although some of these configurations might not be directly detected by $\tilde{H}_{\text {penalty }}$, the minimal energy of $\tilde{H}_{\text {evol }}+\tilde{H}_{\text {penalty }}$, restricted to any of these subspaces, is $\Omega\left(1 / \mathcal{T}^{3}\right)$, and since $\tilde{H}_{\text {init }} \geq 0$ and $\tilde{H}_{\text {final }} \geq 0$ act on the data register and thus within the subspace, they do not affect this lower bound. By multiplying $\tilde{H}_{\text {penalty }}$ by $\mathcal{T}^{2}$, we can boost this to $\Omega(1 / \mathcal{T})$, which will be sufficiently above the low-lying eigenstates in $\mathcal{K}_{0}$.

On the subspace $\mathcal{K}_{0}, \tilde{H}_{\text {penalty }}$ vanishes and we can proceed as before: We choose an initial classical configuration $\boldsymbol{a}$ of the data register and consider the system on the resulting subspace span $\left\{\left|\chi_{0}\right\rangle, \ldots,\left|\chi_{\mathcal{T}}\right\rangle\right\}$. There, it is described by a Hamiltonian very similar to (3), except for minor differences in the implementation of $\tilde{H}_{\text {init }}$ : While $\tilde{H}_{\text {final }}$ can well be applied in the very last $\tau$-time step on the rightmost data qubit and thus give the same penalty term $B=0,1$ in (3), the penalties enforcing properly initialized ancillas can appear in the first $M \tau$-time steps; i.e., a penalty $\mathcal{T}$ can show up in any of the first $M$ diagonal entries of (3). Since this only increases $\theta$ in (5) and thus the gap, we obtain the same spectral properties as before (but we also have to use the lemma for the "yes" instances). Note that, in particular, all energies are well below the energy of any subspace $\mathcal{K}_{S}$ with illegal configurations.

Let us now investigate the entanglement structure of the low-lying eigenstates that are of the form (4), but in the 1D encoding. As before, there are two sources of entanglement: On the one side, we have a superposition of all $\tau$-time steps, i.e., of $\mathcal{T}=\operatorname{poly}(N)$ states. Each of them is almost classical, with the only source of entanglement being one Toffoli gate that is performed. As this involves three qubits, each of these states is a superposition of at most 8 classical states, and thus all low energy eigenstates 
of the 1D Hamiltonian can be written as a superposition of $\operatorname{poly}(N)$ classical states. It follows immediately that the ground state can be represented by an MPS with bond dimension $D=\operatorname{poly}(N)$. Note that the bond dimension actually needed is considerably smaller than the number of classical states, since, e.g., the superposition of $O\left(M^{2}\right)$ states that arises from encoding the $t \leftrightarrow t+1$ transition in $O\left(M^{2}\right) \tau$-time steps ranges over two consecutive $t$-time slices and thus $2 M$ sites only.

We have shown that if the 1D QMA construction of [11] is applied to problems from the class NP, the resulting Hamiltonian has a polynomial gap, and the low-lying eigenstates are MPS. Let us now see what this implies for the difficulty of finding ground states of 1D systems. First, let us encode the verifying circuit for an NP-complete problem in the Hamiltonian. Thereby, we obtain a polynomially gapped Hamiltonian for which the ground state manifold is spanned by MPS, and for which finding a ground state - or even an approximation within an accuracy sufficiently smaller than the gap-is NP hard. Note that obtaining the MPS representation of the ground state is indeed stronger than just deciding the NP problem itself, since from it one can efficiently extract the satisfying assignment.

Let us now construct Hamiltonians with a unique ground state. In order to have a unique ground state for all instances, we have to restrict to problems that have both unique proofs for "yes" and unique disproofs for "no" instances, or more formally problems in NP $\cap$ coNP with unique proofs (coNP is the class of problems where "no" instances can be disproven). Although this is likely to be a smaller class than NP, it still contains interesting hard problems [12]. In particular, finding the prime decomposition of a number corresponds to a problem in this class: It always exists, it is unique, and since primality testing is in $\mathrm{P}$, it can be efficiently checked whether a given decomposition is indeed the prime decomposition. The resulting Hamiltonian has an MPS as its unique ground state, a polynomial gap above it, it is frustration-free since there is always an accepting input, and approximating the ground state by an MPS is at least as hard as factoring; in particular, the prime decomposition can be read off the MPS.

Beyond these implications, our results also provide strong evidence against the existence of a certifiable version of DMRG. The idea behind certifiability is that even if an algorithm does not always converge to the ground state, in case it succeeds this can be certified. To see why no variational method over MPS can be certifiable, take again an NP-complete problem encoded in a 1D Hamiltonian. On the one hand, for any "yes" instance success can be readily checked as the Hamiltonian is frustration-free. However, if it were possible to certify the ground state for "no" instances, this would be a way to provide an efficiently checkable certificate that one is facing a "no" instance for any problem in $\mathrm{NP}$, proving $\mathrm{NP}=$ coNP, which is considered unlikely.

Finally, since the Hamiltonian for a "yes" instance is always frustration-free, one cannot even hope for a DMRG algorithm that only works for frustration-free Hamiltonians. Otherwise, run the algorithm on any instance of an NP problem and check the energy of the state returned: for a "yes" instance, the Hamiltonian is frustration-free and thus the energy is 0 , whereas "no" instances can be easily detected due to their larger ground state energy. Thus, the existence of such an algorithm would allow one to solve NP-complete problems in polynomial time.

Note that our construction to obtain NP hard ground state problems with simple spectral properties will work for any QMA scheme, as all of them are based on Kitaev's original construction. For two-dimensional Hamitonians this is less interesting since there exist hard classical Hamiltonians; however, it might be interesting to apply it to the translational invariant constructions of [13].

We thank S. Bravyi, A. Kay, and T. Osborne for helpful discussions. N. S. thanks the Erwin Schrödinger Institute in Vienna, where parts of this work were carried out, for their hospitality. This work was supported by the EU (SCALA) and the DFG (MAP).

[1] S. R. White, Phys. Rev. Lett. 69, 2863 (1992).

[2] U. Schollwöck, Rev. Mod. Phys. 77, 259 (2005).

[3] F. Verstraete, D. Porras, and J. I. Cirac, Phys. Rev. Lett. 93, 227205 (2004).

[4] M. B. Hastings, J. Stat. Mech. (2007) P08024.

[5] J. Eisert, Phys. Rev. Lett. 97, 260501 (2006).

[6] D. Aharonov and T. Naveh, arXiv:quant-ph/0210077.

[7] A. Y. Kitaev, A. H. Shen, and M. N. Vyalyi, Classical and Quantum Computation (American Mathematical Society, Providence, RI, 2002).

[8] Note that the idea to use classical verifiers, corresponding to the class MA, has already been used in [9] to show that finding ground states of so-called stoquastic Hamiltonians is MA hard. However, while this makes the Hamiltonian stoquastic, it does not lead to a simple spectrum or weakly entangled proofs, since the outcome is nondeterministic and $|+\rangle$ ancillas are being used to obtain randomness. Note that our Hamiltonians are also stoquastic if one allows for a direct implementation of the Toffoli gate.

[9] S. Bravyi, D. P. DiVincenzo, R. I. Oliveira, and B.M. Terhal, arXiv:quant-ph/0606140.

[10] $O, \Omega$, and $\Theta$ denote lower, upper, and exact bounds on the asymptotic scaling, respectively.

[11] D. Aharonov, D. Gottesman, S. Irani, and J. Kempe, arXiv:0705.4077.

[12] C.M. Papadimitriou, Computational Complexity (Addison-Wesley, Reading, MA, 1994).

[13] A. Kay, Phys. Rev. A 76, 030307(R) (2007); K. Vollbrecht and I. Cirac, Phys. Rev. Lett. 100, 010501 (2008). 